\title{
The Application of Medical Virtual Simulation Technology in
}

\section{Medical Education}

\author{
Xiaoping Zhu, Xiaohong Li, Ke Yu* \\ Sichuan Medical University, Luzhou 646000, China \\ ${ }^{*}$ Corresponding author
}

Keywords: Virtual simulation; medical education; clinical skill training

\begin{abstract}
The resulting background of medical virtual simulation technology and the advantage of application in clinical medicine training are analyzed, and emphatically introduce and discuss application research of virtual simulation technology in clinical skills training and assessment.
\end{abstract}

\section{Introduction}

Computer virtual simulation is an emerging high and new technology in recent years; it is an advanced simulation means which can beyond physics and time and space limitations. By using computer technology, computer virtual simulation generates a two-dimensional or three-dimensional space environment like the real reality, the user through the use of various interactive devices; interact with the entity in the virtual environment to produce vivid interactive situation simulation and information exchange. In the development and promotion of computer virtual simulation technology, medical simulation technology and education emerged and developed rapidly. Medical simulation center are widely used in medical teaching, training and assessment field. Medical simulation education is by using medical simulation technology to create the simulated patients and simulated clinical scenarios, instead of real patient to carry on education methods of clinical teaching and practice.

Traditional clinical skill training mode is under the guidance of the superior physician, the patients are as direct practice object, train students to collect medical history, physical examination, clinical diagnosis and treatment and so on. But "occupational physicians act" is introduced, self-protection awareness of patients' continue to enhance and expectations of the medical results rise, which result in opportunities for clinical practice activity and skills training significantly reduced, even cannot reach the training requirements, which became problem troubled modern medicine educational development. With the increase in the number of medical students, training equipment, internship site is tense; contradictions are more obvious, so the students' hands-on opportunities reduce; compared with the theoretical teaching, lack of clinical skills teaching conditions become is more prominent. Meanwhile, we still followed the teacher-led teaching mode. The clinical basic training in curriculum set and teaching content is deficient, while lack of training of scientific thinking and innovation capability, the teaching assessment system also lacks assessment of critical thinking and innovation capability, comprehensive capabilities assessment cannot guarantee objectivity, impartiality. To ensure the quality of higher medical education, make our medical students not only have solid theoretical foundation after graduation

and also have solid clinical skills, it is necessary to explore new training model to increase training resources. Therefore, how to make medical students perform standardized, scientific and 
institutionalized clinical basic skills training, which has become one of the topics of global medical education reform research. Emergence of medical virtual simulation technology will dramatically change the traditional teaching ideas and thinking ways, it will undoubtedly have a profound impact on modern medical education. Because virtual reality is realistic and funny, it does not learn by rote, which not only make students complete the learning task easier and enjoyable, but also can save more time, you can learn more learning content at the same time. Virtual reality has a broad development and application prospects. Application of this technology, which makes traditional medical education model has changed from the following three main areas: Innovation: provide new teaching methods, virtual surgery, virtual diagnosis, virtual care, making students learn in a highly immersive simulation environment. Breakthrough: creating new learning scenes, completely breaking space constraints. Save: application of virtual medical devices, virtual ill, virtual ward will save expensive equipment funds.

\section{The advantages of medical simulation systems}

Medical simulation system can be divided into basic anatomical models, partial functional training models, computer interactive model, virtual training systems and physiology-driven simulation system. According to the scale, medical simulation systems can be divided into clinical skills simulation laboratory, medical simulation centers and simulated hospital.

The advantages in clinical skills training: (1) can simulate some rare, high-risk, cases must be rapid and correct treated, repeatedly carry on relevant clinical skills training. It can follow teachers and students' schedule, according to the need to stop, slow down and re-operation, it has convenient time, repeatability and other features; (2) training in various difficulty and stage can be performed in simulation system, but also can target a certain pathology simulate different causes. (3) the patient without any risk, allowing the error. When using the simulation system, it can be regarded as completely real patients, students can go wrong, but not cause harm to the patient, the error will be corrected in time, it is favorable to enhance memory, meanwhile, training students to use the peaceful mind to treat patients, master operating essentials, improve proficiency. (4) train students teamwork ability. You can take advantage of simulation teaching, teamwork training is carried on in the simulation system, commonly treat patients, and develop their spirit of teamwork. (5) the overall quality and skills of medical students is made OSCE evaluation, assessment.

In summary, medical simulation system has repeatability, training relative authenticity, training content normalization, means richness, training adjustability, patient safety, operational corrective, etc., it help medical students master basic operation specification, clinical skills training of physicians and specialist and some new technology of clinical practice has unique advantages, and make up for the lack of teaching diseases, inadequate treatment operation numbers, thus lower costs, fully improve clinical basic skills of medical students, occupational physicians and specialist physicians, as well as the ability to develop their clinical thinking and innovation.

\section{The role of medical simulation education in clinical skills training}

Since the simulation teaching has unparalleled advantages of traditional teaching mode, some domestic medical colleges learn from foreign mode, by using modern educational technology, clinical skills simulation training center are established. 


\section{The role in the medical students' clinical skills training}

The medical simulation education can make basic teaching; clinical teaching and clinical practice interpenetrate. Students can use standardized patients and simulation systems, to develop their love, understanding, help for the patient in the course of practice operations, train students to contact the patient and the ability to communicate with patients, understand patient's physiological and pathological mechanisms with physiological drive simulation system, clinical skills training and assessment is carried on. The clinical basic skills that students must master are integrated into practice teaching, students' comprehensive clinical operation skills and clinical comprehensive thinking ability as a whole. The clinical skills operation and simulation treatment are integrated to apply in clinical comprehensive skills assessment, assessment item and examination mode are made, assessment indicators and scoring criteria are detailed, establish a scientific and fair evaluation system to assess and evaluate the clinical treatment ability students and clinicians.

\section{The role of clinical skills training after graduation}

At present, physician and junior physician in general hospital, the most is composed of young physicians get master's or doctor's degree just now. They go through postgraduate examination, doctoral examination, do research and many years training, usually with solid theoretical basis, higher foreign language level. Because of long-term clinical detachment, lack of practical training, the operation that should master cannot be completed independently. Therefore, we can use the hospital simulation training centers, making these young physicians strengthen the practice, increasing the opportunity to exercise operations. For example: endotracheal intubation, defibrillation, ventilator connection debugging, thoracic drainage and other emergency treatment. At present, many general hospitals most established simulation training center for new admissions physician, using simulation training center for intensive training and assessment, and obtain good results. By using simulation aids, physiological drive simulation systems and devices in clinical practice, for example, monitors, defibrillators, ventilators, etc., clinician training is held twice a year, strengthen basic clinical skills of teachers, it played a very good role on ensuring students' clinical practice education.

\section{The role in the each professional clinical skills training}

\section{High simulated emergency training in first aid medicine}

Emergency medicine is a high-risk subject, it not only requires physicians have emergency medicine knowledge and proficiently master various emergency rescue skills, but also should have a keen observation sense, and ensure every decision, every measure that medical staff made is fast and effective, By using high-analog simulation system for training provided by modern simulation medical technology, it can simulate the human real body's physiological and pathological characteristics, it shows clinical signs consistent with logic, and show physiological parameters by simulating monitoring, and make due response treatment measure. Therefore, in basic skills training of emergency medicine, the application of high simulation person, it can simulate the evolution process of clinical disease of organ dysfunction, for example: cardiopulmonary resuscitation in advanced life support and respiratory failure, circulatory failure and batting training of other acute critical illness. 


\section{Simulated teaching in clinical skills training of anesthesiology specialty}

The feature of basic skill of anesthesia specialty is strong specialty features, it directly related to the medical effects and safety; it must have sufficient proficiency to meet the clinical needs. Currently, some domestic institutions establish anesthesia and resuscitation skills training room, the anesthesiologist through simulation system can simulate visits before surgery, anesthesia induction of surgical patients, intraoperative maintenance, anesthesia monitoring, and postoperative recovery and so on. In the basic skills training of anesthesia specialty of medical students and junior physician, it requires teachers have rich clinical experience, the operation skill and simulation training systems are proficient. It is necessary to formulate a detailed lesson plans and training guide, meanwhile, it should be good at design the training process, review and summarize.

\section{The virtual simulation of endoscope and training system}

Foreign scholars have explored the effectiveness of bronchoscopy simulation training system; the research confirms that the use of bronchoscopy virtual simulation training system, beginners' skills has been significantly improved. Bronchoscopy simulation training systems are divided into two types: high simulation computer electronic and low simulation mechanical inanimate airway model. Use bronchoscopy simulation training system can improve the effectiveness and accuracy of the operating procedure; reduce damage of the airway wall, meanwhile, improve beginners' interest and satisfaction. Besides the bronchoscope simulation systems, but also includes other endoscopic simulation systems, such as laparoscopy, gastrointestinal endoscopy and so on.

The role in clinical skills training in obstetrics and gynecology department, some domestic medical school have established simulation lab of obstetrics and gynecology, created a high simulated delivery room and make childbirth education, train students midwifery skills and treatment and cooperation of dystocia. By using computer abdominal examination of pregnant women models can made obstetric examination, auscultation of fetal heart tones, pelvic measurements. In the simulation gynecological surgeries, through advanced gynecological examination model, a variety of gynecological skills can be carried out.

\section{The limitations and prospects of medical simulation education in clinical skills training}

Medical simulation technology can solve some problems that exist in the current medical education, significantly improve the level of medical education; it has been universally recognized by medical education workers. But it is impossible to simulate all the clinical courses, the basic signs of many patients are unable to reflect. Current medical simulation systems most simulate symptoms, and signs are concentrated in the circulatory system, nervous system, respiratory system and the urinary system, and trainee must have adequate basis and clinical knowledge before the training, otherwise, it will not achieve the desired effect. Simulation teaching relies on clinical theory and practical experience of designers, it is currently impossible to exceed the understanding level of predecessors, and may be because the designer is ill-considered and result in insufficient effect. Meanwhile, medical simulation teaching is a high-cost item,

Because medical simulation teaching not only requires advanced computer technology and IT support, but also need the help of the medical model that manufactured by high-tech and advanced materials engineering technology, so the medical simulation teaching is not only a complex system engineering, but also is a project with larger investment, its operation requires a high cost. How to better make the high-end simulation technology applies into teaching, training, assessment, it also need to explore an effective method. Besides, simulation training device or simulation persons are 
now at the development phase of science and technology, there are technical limitations, there are a lot of limitations, and simulation teaching is still in a phase of continuous improvement. Its design, application has some limitations, which cannot completely replace the clinical and medical practice, it should be used with the theory teaching, practice exercises, so as to train medical personnel with comprehensive quality.

Clinical skill teaching is the key to medical education, how to effectively improve the clinical skills in teaching is the key to practice teaching reform of higher medical education. Simulation teaching is becoming an important aspect of domestic medical education reform, with the continuous development of medical simulation technology; it will have a wider range of applications in the frontier area that require medical staff with higher operation skill. From the perspective of broad education, with awareness of the importance of medical simulation and the continuous development of simulation technologies, the simulation technology and equipment are rationally allocated, clinical skills laboratories which can run professional management operation are established, which are the development trend of medical simulation education.

\section{References}

[1] Z. G. Pan, X. J. Jiang, M. M. Zhang and J. Y. Shi, Distributed Virtual Environment: an overview, Journal of Software. 4, 11 (2000) 461-467.

[2] H. Chen, F. M. Chen, Research on VRML-based virtual reality system, Computer Engineering. 7, 27 (2001) 83-85.

[3] H. Jiang, L. Wang and Z. C. Zhang, Application of virtual reality technology in medicine, China Medical Equipment. 8, 23 (2008) 49-52, 71.

[4] J. G. Wang, Research on application of virtual reality technology in practical teaching of higher vocational colleges, Chinese Vocational and Technical Education.23 (2011) 76-80.

[5] J. Zhou, Employing virtual reality technology to improve the education quality of open universities, Continuing Medical Education. 6, 26 (2012) 39-41. 